\title{
A Roadmap to Mitigation Techniques : Bedrock for Atmospheric Turbulence
}

\author{
Krina Patel
}

U and PU. Patel Department of Computer Engineering, CSPIT, CHARUSAT, Changa, Gujarat, India Email : krinap003@gmail.com

Dippal Israni

U and P U. Patel Department of Computer Engineering, CSPIT, CHARUSAT, Changa, Gujarat, India

Email : dippalisrani.ce@charusat.ac.in

Dweepna Garg

Department of Computer Engineering, DEPSTAR, CHARUSAT, Changa, Gujarat , India

Email : dweepnagarg.ce@charusat.ac.in

-ABSTRACT-

A momentous distortion of the scenes captured through long distances is a result of atmospheric turbulence due to changes is air refractive index. A series of different methods have been proposed to mitigate the consequences of atmospheric turbulence, enhancing the performance of imaging systems. This paper comprehends an overview to various techniques to alleviate these turbulence based on different approaches along with descriptions, working principles and challenges. The existing approaches for air turbulence are fusion based, optical flow based, phase based and latent image based techniques. This paper also describes standard dataset used for resolving turbulence mitigation issue. It also represents new ideas and innovations for mitigation of turbulence. Finally, the performance evaluation parameters such as MSE, PSNR, and SSIM are also discussed.

Keywords -Atmospheric turbulence, Image fusion, Long range video Surveillance,Optical flow

Date of Submission: Jan 10, 2019

Date of Acceptance: Apr 09, 2019

\section{Introduction}

$\mathrm{H}_{\mathrm{i}}$ igher layers of fog and smog into the atmosphere form a barrier for space and air travelers. It limits the visibility and thus affects human activities that rely on clear visibility conditions. Such activities include aircraft operations, shipping and Road traffic[1]. Images and videos captured from distant places, such as groundsurveillance[2] and astronomy[3] face a lot of atmospheric turbulence. This results in a distortion of captured images and video signals. The problems of restoring a clear image from a sequence of turbulence-degraded frames are of high research interest, as the effect of such distortions and blur would significantly degrade the quality of image[4].

Atmospheric turbulence is a natural phenomenon induced from random fluctuations [5] of the air that alters the air refraction index along the light transmission path. The dust particles, fog, haze and revolving aerosols that reflects the light falling on the lens of the camera capturing image or video sequence[6]. Such alteration could be certainly observed at places where the temperature is higher.It include places such as hot road surfaceswhere the solar radiations heats the surface and the air above it making it more warmer and buoyant, deserts, exhausts of jet planes. Consequently, the motion of real moving objects is similar to that caused by turbulence in the scene. It results into induced geometric distortion, additive noise and a space and time varying blur[3]. The turbulence can be demonstrated as:

$$
T_{n}=D_{n} B_{n} I+\epsilon
$$

Where, $T_{n}$ denotes the $n^{\text {th }}$ frame, $D_{n}$ represents the deformation matrix, $B_{n}$ depicts the blurring matrix, $I$ is the Ideal pixel matrix without turbulence and $\in$ denotes the induced noise.

Atmospheric turbulence mitigation techniques are broadly utilized in computer-simulated landings of aircraft, defense [7], long-range surveillance systems[2][8], underwater imaging systems, remote sensing [9] and many computer vision applications[10][11].Effects of turbulence are attained in extreme situations where it is difficult to interpret information behind the distorted layer of turbulence.Traditional image registration approach lags as it is computationally expensive and need post-processing algorithm for sharpening the image. A non-registration based Sobolev Gradient and Laplacian (SGL) algorithm[12] removes turbulence but results in ghost artifacts in moving objects.

This paper mainly focus on various existing turbulence mitigation techniques along with turbulent dataset description and parameters for evaluation of turbulence. The methods are based on different approaches of image and video processing. 


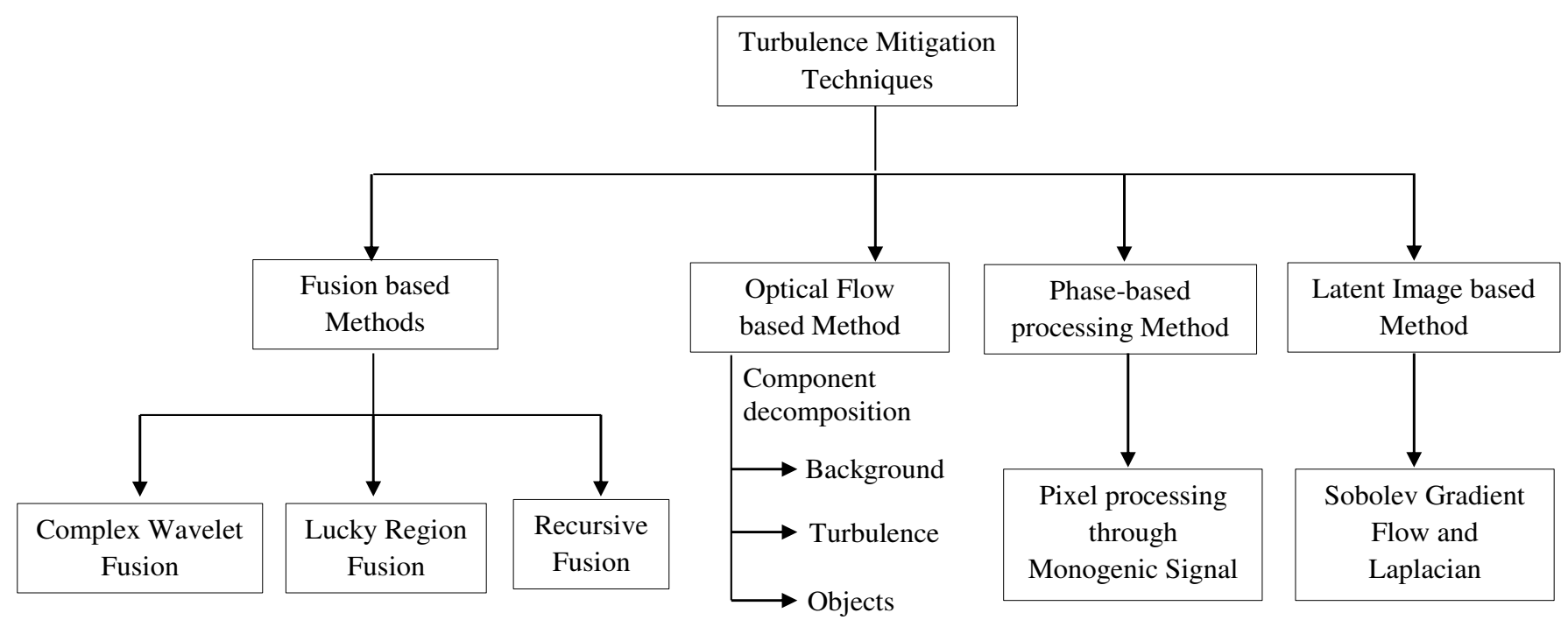

Fig 1. Different techniques to mitigate Atmospheric Turbulence

\section{Various techniques to evaluate turbulence}

Generally, existing turbulence alleviation algorithms for imaging systems to restore turbulence-free high quality video sequences is broadly based on different approaches. The techniques are showcased in Fig 1.

\subsection{Image Fusion based Methods}

Image Fusion is a method through which valuable information from a number of image source are selected and combined to form a new image. Due to shift invariance, multi-scale properties and dimensional selectivitythe DT-CWT is extensively used for image fusion. DT-CWT is used to overcome the drawbacks to the traditional wavelet transform. Its complex value filtering decomposes the real and complex signals into respective real and imaginary parts in transform domain.

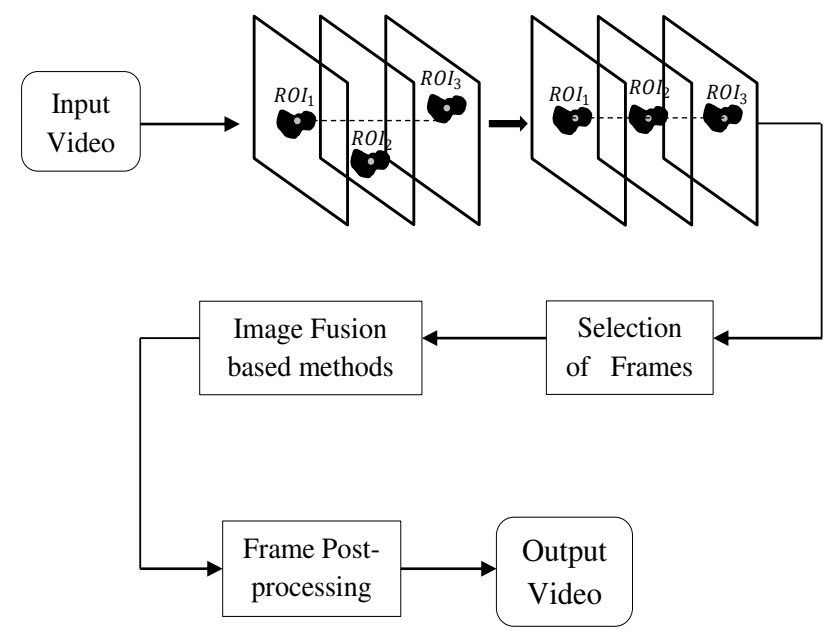

Fig 2. Block diagram of Fusion based techniques
Prior to image fusion various approaches are considered such as ROI (Region Of Interest) Alignment [3] [7], Temporal

Frame Registration, to find matching features between groups of adjacent images.As capturing videos in the presence of atmospheric turbulence will seamlessly cause the ROIs in each frame to be misaligned, their alignment is a crucial step. The size of the ROIs are detected and evaluated as one of the factor for frame selection, along with sharpness and intensity similarity.

Finally, the technique is followed by the fusion based methods that attempt to align objects temporally in low turbulence conditions and for a small movement of camera[14]. The fusion here is performed in Dual Trees Complex Wavelet Transform (DT-CWT) domain as it provides directional selectivity, multi-scale properties and near shift invariance. These methods are not efficient in the case of large moving objects or video sequences with high turbulence. It also fails to solve moving camera sequences.

\subsection{Optical Flow based Methods}

Optical flow based mitigation techniques are designed with less noise and high resolution. It reduces the impact of atmospheric turbulence by reducing the resultant geometric distortion and blur. It also preserves the important information in the frames. The weighting cues based bon the optical flow are used to separate out true objects motion from turbulent motion[15]. These method is solely used to simultaneously reduce the turbulence accompanied by preserving moving objects which may be of great interests. It can be achieved by separating different components of an image sequence as in fig 4 into background, scintillations and the objects[16]. 
These decomposition can be formulated as:

$$
F=B+O+E
$$

Where, $\mathrm{F}$ denotes the given series of frames $\left(I_{1, \ldots,} I_{n}\right)$ acquired from a stationary camera, B, O, E[17] represents pixel matrices for background, object and error or turbulence respectively.

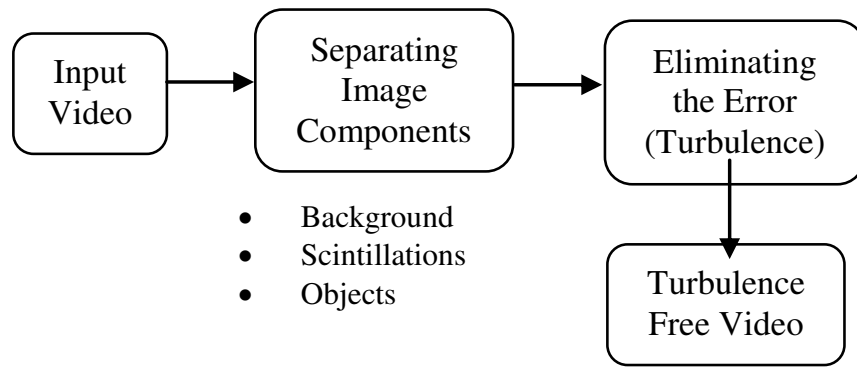

Fig 3. Block diagram of Optical Flow based techniques

This decomposition is mainly corresponding to the inherent properties of the components. The background scene is presumed to be static, hence it consists of linearly correlated elements that are a part of a low rank matrix[18]. The turbulence component[17] can be captured by the Forbenius norm attained from a matrix by piling the columns. The moving object components[19] are best taken by limiting the non-zero records desirable for finding outliers. The object is any video sequence is assumed to occupy a smaller amount of space as compared to the entire frame pixel.

This method is composed of :1) solvingthe problem of simultaneous moving object detection and turbulence mitigation by a low-rank optimizationbased matrix decomposition[20]. 2) A turbulence model based on motion and intensity, where the Lagrangian particle advection Framework is used for motion distribution.3) tohandle long sequences without discontinuities a force component in the particle advection framework is enacted.

\subsection{Phase based Processing Techniques}

The influence of turbulence on imaging seemto be a pixel intensity scintillation and pixel temporal oscillation. The degraded effects of atmospheric turbulence can be transformed to analyze the local amplitude and local phase[21] of the monogenic signal[22] according to the phase-based image processing techniques. The phaseinformation preserves the inherent features of the image by retaining the phase information of the signal.

It deals with simultaneously moving object preservation and turbulence-distorted video stabilization. The monogenic signal comprises monogenic amplitude and phase, they can be effectively used to represent degraded image structure and motion information and are insensitive to image contrast. These method consists of two stages: attaining the stable background frames and restructuring the stabilized video preserving moving object. The first stage comprises of three steps: 1) series of input videoframeis represented as a signal where it consists of the phase and amplitude of the signal. 2) temporal filteringof the monogenic phase and amplitude3) the restoration of the frames corrected background. The second stage involves three steps: 1) the creation of bristly masks in video for moving objects. 2) Refinement of Mask through temporal exploration of the signal. 3) The video is restored along withpreservation of the object in motion.

\subsection{Latent Image based Processing Technique}

It involves a simple and numerically stable method that unwraps the video sequence and reconstructs a sharp latent image. This method employs Sobolev gradient for individual frame sharpening and Laplacian operator[12] to alleviate temporal diffusion on the input video at the same time. It is assumed that the image is repeated as a customary in Fourier-based approach. It projects the linear operators onto the eigen functions of the Laplacian yielding a satisfactory output video. Moreover, The image deblurrring is performed that results into more naturally sharp image alhough oscillations at the boundary still remains. Hence, The main idea for annihilating the effects of atmospheric turbulence is to stabilize these oscillations along with sharpening individual video frames.

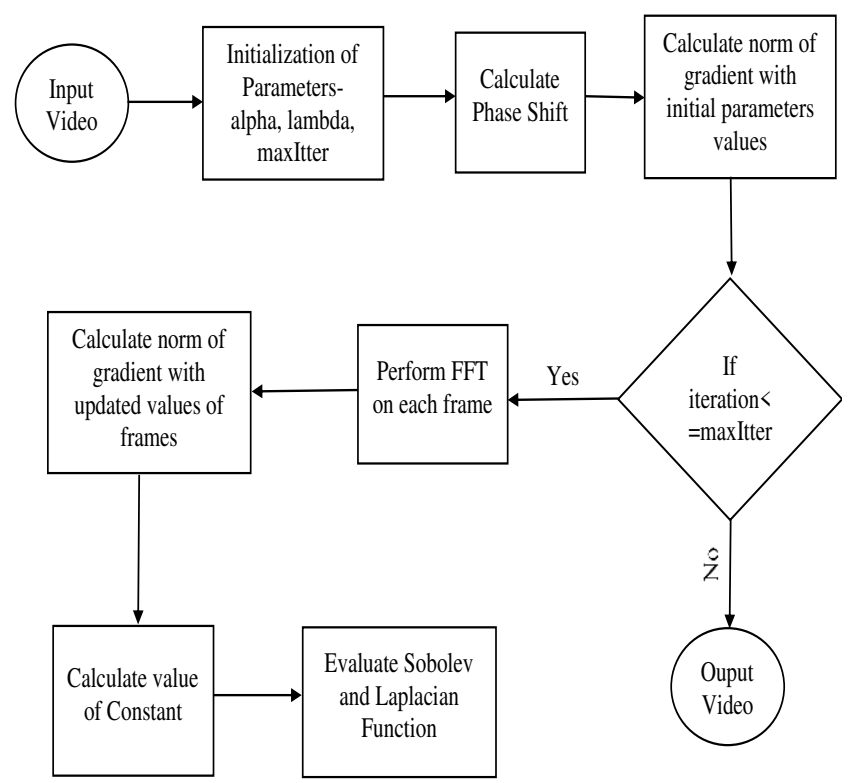

Fig 4. Block diagram of Latent Image based processing technique

Parameter taken for the approach is $\alpha$ spatial sharpening factor for each frame, $\lambda$ for temporal smoothing, $\mu$ for balancing between the spatial sharpening and temporal smoothing. Calculation of thePhase shift is the horizontal distance or displacement of a point from its original position. The horizontal movement maybe to the right or left depending on the motion flow of turbulence.Calculation of norm of the gradient refers to the norm with an initial value of the frame and the norm of the updated value of frame whose value is calculated using FFT function. Followed by the calculation of the constant value. Constant value computation stops when the ratio of numerator and denominator is equal to sharpness factor $\alpha$. 
The function uses the previous and next frame with respect to the current frame and performs SGL function on it thus improving the frame result by sharpening and temporal smoothing factor of it. After the calculation, it takes the simple average of the frame and generates a new frame by adjusting the parameter and distorted objects. The geometric distortion is corrected by matching the shift map of consecutive previous and next frame as per the window size. Calculation of average corrects the blur effect on the frame thus sharpens the frame. Turbulence free video sequence generated. Using this approach it reduces the oscillation caused by turbulence and also corrects the blurred frame.

\section{Dataset Description}

The dataset used in this experiment is from the state of art dataset- OTIS (Open Turbulent Image Set)[14] available online. These datasets are standard datasets containing sequence of image frames induced with turbulence which has been used for experimenting and simulation. There are 100 image frames taken for processing. The below mentioned are the detail descriptions of the dataset used:

Table 1 : Information of dataset

\begin{tabular}{|l|l|l|l|l|}
\hline $\begin{array}{l}\mathrm{Sr} \\
\text { No. }\end{array}$ & Video & $\begin{array}{l}\text { Dataset } \\
\text { Provider }\end{array}$ & Band & $\begin{array}{l}\text { Dimensions } \\
\text { (Height*Width) }\end{array}$ \\
\hline 1 & Door & OTIS & Visible & $520 * 520$ \\
\hline 2 & Pattern 5 & OTIS & Visible & $113 * 117$ \\
\hline 3 & Car 1 & OTIS & Visible & $200 * 200$ \\
\hline
\end{tabular}

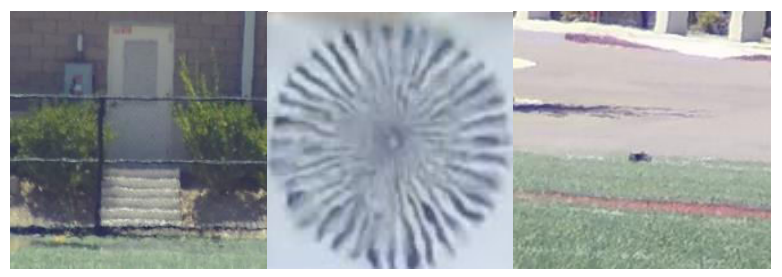

Fig 5. (a) Door[14] $\quad$ (b) Pattern 5[14] $\quad$ (c) Car 1[14]

\section{Simulation Results}

The above mentioned turbulence removal algorithms alleviates the turbulence from the sequence of images and videos.. In case of video sequences, it compares

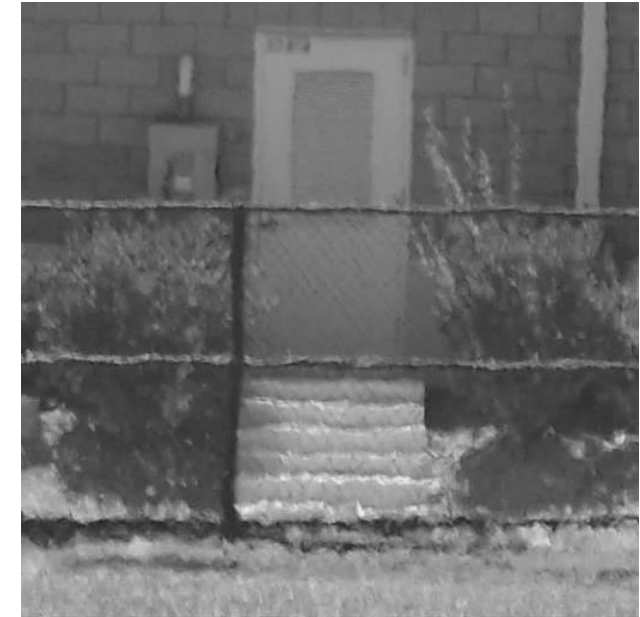

Fig 6. Original image frame with turbulence

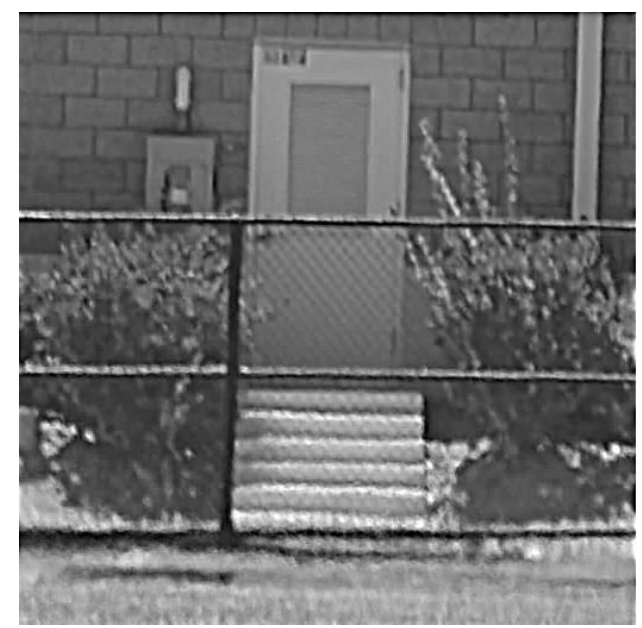

Fig 7. Processed image frame after turbulence removal

with the consecutive frames and hence removes turbulence. Fig 6 and Fig 7 depicts the respective $16^{\text {th }}$ original and processed frames from the door dataset video. The video is converted to gray scale and hence the output is showcased.

\section{Parameters for Evaluation}

Quality assessment of the frames is used to measure the amount of image degradation by comparing it to a reference frame that is distortion free. But, In case of video sequences affected by turbulence there does not exist any reference frame to compare with, as all frames consist of turbulence motion. Hence, the assessment is done temporally by comparing the adjacent frames of a video sequence and computing the parameter values. Theparameters used for measurement can be Mean Square Error (MSE), Peak Signal to Noise Ratio (PSNR), Multi Scale Structured Similarity (MS-SSIM)[9].

1) MSE: It is the measure of the average of the square of the errors[23][24].It is mathematically formulated as:

$$
M S E=\frac{1}{m n} \sum_{x=0}^{m-1} \sum_{y=0}^{n-1}[I(x, y)-N(x, y)]^{2}
$$


Where I is the image intensity and $\mathrm{N}$ is the noise present in the image frame.

2) SSIM:It is based on the visible structure in the image.It is a metric that quantifies image quality degradation by splitting the measurements of similarity based on various windows of an image[23]. The measure between two windows with same dimensions is given by:

$$
\operatorname{SSIM}=\frac{\left(2 \mu_{m} \mu_{n}+w_{1}\right)\left(2 \sigma_{m n}+w_{2}\right)}{\left(\mu_{m}^{2}+\mu_{n}^{2}+w_{1}\right)\left(\sigma_{m}^{2}+\sigma_{n}^{2}+w_{2}\right)}
$$

Where, $\mu_{m}, \mu_{n}$ and $\sigma_{m}^{2}, \sigma_{n}^{2}$ are means and variances of windows $\mathrm{m}$ and $\mathrm{n}$ respectively, $\sigma_{m n}$ is the covariance of $\mathrm{m}$ and $\mathrm{n}$.

3) PSNR: It is used to compute the superiority of reenactment of the image. A higher assessment of PSNR indicates the better feature of image reconstruction[25]. It is stated as:

$$
P S N R=20 . \log _{10}\left(M A X_{i}\right)-10 \cdot \log _{10}(M S E)
$$

As in equation (5) $M A X_{i}$ is the maximum possible pixel information of the image.

\section{Conclusion}

The different techniques of mitigation of atmospheric turbulence from video sequences is discussed and summarized in this is paper. Most of the available techniques are applicable only for low or medium turbulence due to non-availability of appropriate distortion functions. Additionally, the existing methods do not consider independent turbulence and object motion. However, at times real motion of object gets distorted in order to remove the turbulence. It has been observed that most algorithms showed optimum results when input video is a static scene, but is not effective for moving objects in the scene. Traditionally, there are very few articles that deal with moving object detection and preservation under turbulence. Nowadays, one of the most emerging research topicis the moving object preservation in the presence of atmospheric turbulence.

\section{ACKNOWLEDGEMENT}

The authors would like to thank Charusat Space Research and Technology Center (CSRTC) for providing required resources and platform to carry out research work.

\section{REFERENCES}

[1] B. Fishbain, L. P. Yaroslavsky, and I. A. Ideses, "Real-time stabilization of long range observation system turbulent video," J. Real-Time Image Process., vol. 2, no. 1, pp. 11-22, Oct. 2007.

[2] J. Khan, K. Khan, and W. Goodridge. "Multi-Criterion Decision Making and Adaptation for Multi-path Video Streaming in WSNs." International Journal of
Advanced Networking and Applications, vol. 9, no. 2 pp. 3376-3381, Sep 2017.

[3] V. Chaudhary, "Literature Review: Mitigation of Atmospheric Turbulence Impact on Long Distance Imaging System with Various Methods," vol. 3, no. 12 , p. 5, 2012.

[4] N. Anantrasirichai, A. Achim, N. G. Kingsbury, and D. R. Bull, "Atmospheric Turbulence Mitigation Using Complex Wavelet-Based Fusion," IEEE Trans. Image Process., vol. 22, no. 6, pp. 2398-2408, Jun. 2013.

[5] N. Anantrasirichai, A. Achim, and D. Bull, "Atmospheric turbulence mitigation for sequences with moving objects using recursive image fusion," ArXiv180803550 Cs, Aug. 2018.

[6] A. Singh and R. K. Singh, "A Survey on Restoration Techniques of Atmospheric Turbulence Blur Image," vol. 4, p. 9.

[7] M. Anum, M.Shahid, and M. Sharif. "Content-Based Image Retrieval Features: A Survey." International Journal of Advanced Networking and Applications, vol. 10, no. 1, pp. 3741-3757, 2018.

[8] E. Chen, O. Haik, and Y. Yitzhaky, "Detecting and tracking moving objects in long-distance imaging through turbulent medium," Appl. Opt., vol. 53, no. 6, p. 1181, Feb. 2014.

[9] R. Kumar, M. Purohit, D. Saini, and B. K. Kaushik, "Air Turbulence Mitigation Techniques for LongRange Terrestrial Surveillance," IETE Tech. Rev., vol. 34, no. 4, pp. 416-430, Jul. 2017.

[10] P. Scholar, "Restoration of Turbulence Images using Complex Wavelet-Based Fusion," p. 4.

[11] P. Ochs, J. Malik, and T. Brox, "Segmentation of Moving Objects by Long Term Video Analysis," IEEE Trans. Pattern Anal. Mach. Intell., vol. 36, no. 6, pp. 1187-1200, Jun. 2014.

[12] A. L. Bertozzi, S. Soatto, S. H. Kang, and Y. Lou, "Video stabilization of atmospheric turbulence distortion," Inverse Probl. Imaging, vol. 7, no. 3, pp. 839-861, Sep. 2013.

[13] A. Aruna and A. Rajani, "A REVIEW ON MITIGATION OF ATMOSPHERIC TURBULENCE WITH IMAGE FUSION IN VISUAL SURVEILLANCE," vol. 2, no. 10, p. 4.

[14] M. Hemarlin, "Complex Wavelet-Based Fusion Involved In Atmospheric Turbulence Mitigation,” p. 6, 2014

[15] D. Li, "Suppressing atmospheric turbulent motion in video through trajectory smoothing," Signal Process., vol. 89, no. 4, pp. 649-655, Apr. 2009.

[16] O. Oreifej, X. Li, and M. Shah, "Simultaneous Video Stabilization and Moving Object Detection in Turbulence," IEEE Trans. Pattern Anal. Mach. Intell., vol. 35, no. 2, pp. 450-462, Feb. 2013.

[17] P. E. Robinson and A. L. Nel, "Foreground segmentation in atmospheric turbulence degraded video sequences to aid in background stabilization," J. Electron. Imaging, vol. 25, no. 6, p. 063010, Nov. 2016. 
[18] A. Elkabetz and Y. Yitzhaky, "Background modeling for moving object detection in long-distance imaging through turbulent medium," Appl. Opt., vol. 53, no. 6, p. 1132 , Feb. 2014.

[19] A. Deshmukh et al., "Embedded Vision System for Atmospheric Turbulence Mitigation," in 2016 IEEE Conference on Computer Vision and Pattern Recognition Workshops (CVPRW), Las Vegas, NV, USA, 2016, pp. 861-869.

[20] C. P. Lau, Y. H. Lai, and L. M. Lui, "Restoration of Atmospheric Turbulence-distorted Images via RPCA and Quasiconformal Maps," ArXiv170403140 Cs, Apr. 2017.

[21] K. Ito and T. Aoki, "Phase-based image matching and its application to biometric recognition," in 2013 Asia-Pacific Signal and Information Processing Association Annual Summit and Conference, Kaohsiung, Taiwan, 2013, pp. 1-7.

[22] C. Zhang, F. Zhou, B. Xue, and W. Xue, "Stabilization of atmospheric turbulence-distorted video containing moving objects using the monogenic signal," Signal Process. Image Commun., vol. 63, pp. 19-29, Apr. 2018.

[23] F. Alvarez, L. Tahir, N. Ferrante, A. Tarter, and M. Fortman, "Image Processing and Restoration under Atmospheric Turbulence," p. 59.

[24] D. Kheni, T. Italiya, D. Isarani, and D. Karthick, "A novel blind approach for image restoration using adaptive kurtosis based deconvolution," in 2017 2nd IEEE International Conference on Recent Trends in Electronics, Information \& Communication Technology (RTEICT), Bangalore, 2017, pp. 957962.

[25] T. Caliskan and N. Arica, "Atmospheric Turbulence Mitigation Using Optical Flow," in 2014 22nd International Conference on Pattern Recognition, Stockholm, Sweden, 2014, pp. 883-888.

\section{AUTHOR'S PROFILE}

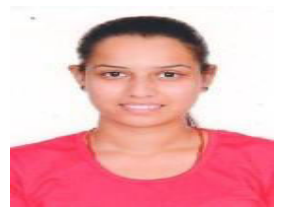

Krina T. Patel is pursuing her Master of Technology degree in Computer Engineering from CSPIT, CHARUSAT, Gujarat (India).She has received her Bachelor of Engineering degree in Information Technology in 2017 from CSPIT, CHARUSAT, Gujarat (India).Her area of interest includes Computer Vision, Machine Learning, Artificial Intelligence, Deep Learning.

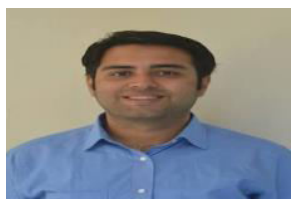

Mr. DippalIsrani is currently working as an Assistant Professor at Chandubhai S. Patel Institute of Technology, CHARUSAT, Gujarat. $\mathrm{He}$ received his Bachelor of Engineering degree in Information Technology from Sardar Patel University, Gujarat (India), and Master of Technology degree in Computer Engineering from
Dharmsinh Desai University, Gujarat (India). His research interests are Computer Vision, Image Processing and Machine Learning.

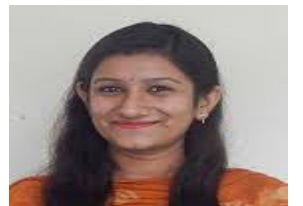

Mrs. DweepnaGarg is working as an Assistant Professor in the Department of Computer Engineering of Devang Patel Institute of Advance Technology and Research, CHARUSAT. She is also pursuing her PhD in the area of Deep Learning since 2015. She has more than 5 years of teaching experience. She is a Gold Medalist in M.E (CSE) from Gujarat Technological University in the year 2014. She secured 8th Rank in B.E in Gujarat University in the year 2011. Her area of interest includes Machine Learning, Deep Learning. 